Nota de investigación

\title{
Asociatividad óptima en las zonas rurales del Ecuador utilizando la teoría de juegos
}

\author{
Gabriela Araujo-Ochoa ${ }^{1 \S}$ \\ Yésica Mayett-Moreno ${ }^{2}$ \\ Katia Angélica Figueroa-Rodríguez ${ }^{3}$ \\ Ezequiel Arvizu-Barrón ${ }^{4}$
}

${ }^{1}$ Universidad Politécnica Salesiana. Cuenca, Azuay, Ecuador. CP. 010101. ${ }^{2}$ Universidad Popular Autónoma del Estado de Puebla. 17 sur núm. 901, Barrio de Santiago, Puebla, México. CP. $72410 .{ }^{3}$ Colegio de Postgraduados-Campus Córdoba-Programa de Innovación Agroalimentaria Sustentable. Carretera Córdoba-Veracruz km 348.5, Congregación Manuel León, Amatlán de los Reyes, Veracruz, México. CP. 94953. ${ }^{4}$ Cátedras CONACYT-Universidad Autónoma Chapingo-Departamento de Sociología Rural. Carretera México-Texcoco km 38.5, Chapingo, Texcoco, México. CP. 56230.

§Autora para correspondencia: garaujo@ups.edu.ec.

\section{Resumen}

La agricultura en Latinoamérica y específicamente en el Ecuador, busca solventar necesidades de autoconsumo y comercialización. En Ecuador, miles de hogares practican la agricultura, la cual representa $70 \%$ de la producción agrícola del país y $60 \%$ de los alimentos que conforman la canasta básica. El objetivo de la presente investigación es proponer un modelo para que los productores puedan trabajar en asociaciones agroecológicas utilizando la teoría de juegos cooperativos, logrando mejorar su productividad, llegando a competir en un dinamismo de costos, oferta, demanda, distancia y tiempo. Se realizó un estudio con enfoque mixto, ya que en la parte cuantitativa se utilizaron encuestas semiestructuradas, análisis descriptivo, así como información secundaria para el modelo matemático numérico de la teoría de juegos cooperativos. En la parte cualitativa, al ser un fenómeno poco estudiado, el tamaño de la muestra fue de 76 agroproductores y el muestreo fue por conveniencia. Por su parte, el diseño de investigación fue no experimental, transversal ya que los datos se recolectaron en un solo momento (2019). La encuesta constaba de 13 ítems, entre ellos la ubicación geográfica, características socioeconómicas del productor y de la producción agrícola, acopio-comercialización y venta del producto, entre otras. La información de la encuesta se analizó mediante estadística descriptiva. Finalmente, utilizando los resultados de las encuestas y bases de datos secundarios se geo-referenció su ubicación para simular dos escenarios comparativos de trabajar o no en asociación mediante el software Matlab. Los hallazgos sugieren trabajar con un máximo de 20 productores por asociación, con número óptimo menor de 10 agroproductores, logrando así reducir las pérdidas de la productividad en $6.4 \%(14.4 \mathrm{~kg})$ de producción.

Palabras clave: asociatividad, comercialización, economía colectiva, planificación agrícola.

Recibido: junio de 2021

Aceptado: julio de 2021 


\section{El enfoque agroecológico}

Este enfoque actúa como una cuenca ideológica de atracción Altier et al. (2012) que drena las inquietudes de una comunidad epistémica en pleno crecimiento y la militancia de los productores movilizados alrededor de la economía solidaria hacia un cauce principal constituido por un paradigma alternativo de bienestar rural. La agroecología es una agricultura alternativa, que toma como modelo de producción a la misma naturaleza, evitando la dependencia de productos químicos para garantizar la conservación y preservación de los ecosistemas naturales y los agroecosistemas.

La agroecología cumple con esta característica e incluso va más allá, al constituirse como una verdadera filosofía de vida (Heifer, 2016). Los productos orgánicos se venden en mercados específicos y a un público sectorizado y gozan de un sobreprecio. Esto se debe a que hoy, la demanda de este tipo de productos supera ampliamente la oferta, además del hecho de que, a través de estrategias de marketing, se los define como productos exclusivos (Hair, 2004). Los productos agroecológicos; sin embargo, se diferencian ampliamente de los orgánicos ya que, aunque tienen formas de producción similar respecto del ambiente y no uso de agroquímicos, persiguen objetivos ampliamente diferenciados.

Estos productos pretenden ayudar a las economías regionales y a los agricultores pobres a subsistir y priorizan el autoconsumo. Muchos productos orgánicos, además, no son de origen local o regional, sino que se producen con técnicas amigables con el ambiente, pero se exportan a otros países o continentes. En este sentido uno de los requisitos de los productos agroecológicos es que tiene que comercializarse en la misma región en que fue producido, tanto para colaborar con la economía regional y seguridad alimentaria local, como para mantener un precio razonable y ser accesible para toda la sociedad (Saal, 2015).

Por otro lado, en el mundo existe un sector campesino con una población estimada en 65 millones, que incluyen 40-55 millones de indígenas que hablan aproximadamente 725 lenguas (Altieri y Toledo, 2011). Con base en estimaciones de hace una década, estos productores campesinos (tamaño medio del predio de 1.8 ha), producen 51\% del maíz, 77\% de los granos y $61 \%$ de las papas que se consume en la región Altieri (2002). Sólo en Brasil, hay aproximadamente 4.8 millones de agricultores familiares (cerca de $85 \%$ del número total de productores agrícolas), que ocupan 30\% del total de tierras agrícolas del país, que controlan alrededor de 33\% de la superficie sembrada con maíz, $61 \%$ de que en virtud de frijoles y de $64 \%$ que, sembrada de yuca, lo que produce $88 \%$ del total de yuca y $67 \%$ de todos los granos.

En Ecuador, el sector campesino ocupa más de 50\% de la superficie dedicada a cultivos alimentarios como el maíz, frijol, cebada y ají. En México, los campesinos ocupan al menos 70\% de la superficie cultivada con maíz y $60 \%$ de la superficie de frijol Toledo et al. (2010). De la misma manera en Ecuador las primeras experiencias agroecológicas se empiezan a desarrollar desde el año 1980, pero fue años después que se comienza a establecer un control sobre la producción orgánica, incorporando los sistemas participativos de garantías (SPG), los cuales nacen como una alternativa a las certificaciones de tercera parte (CTP), también se ha desarrollado la normativa legal a favor de la soberanía alimentaria como es la Ley Orgánica del Régimen de Soberanía Alimentaria (LORSA) y la Constitución de la República (Intriago, 2018). 
En la actualidad en la ciudad de Cuenca-Ecuador, las asociaciones agroecológicas, están formadas por personas del campo quienes a su vez vieron una ventaja al trabajar juntas. A pesar de que la mayor parte de su producción es destinada al autoconsumo, una parte es destinada a la venta de los productos. Lamentablemente motivos como la desconfianza en el trabajo conjunto como asociación, falta de información del manejo de la agricultura agroecológica, la falta de preparación técnica, la falta de competitividad ante productos importados de países cercanos, los bajos ingresos que perciben por sus ventas, así como los costos elevados para poder transportar sus productos a los distintos mercados; han desencadenado algunos problemas en estas asociaciones.

Muchas prefieren trabajar de manera individual a pesar de estar asociados, lo cual ha provocado el desaprovechamiento de oportunidades de crecimiento para mejorar sus ingresos, puesto que organismos como el Ministerio de Agricultura y Ganadería (MAG, 2014), tienen técnicos que les dan capacitaciones. Lamentablemente en su mayoría no asisten a éstas, por lo tanto, no pueden conocer nuevas técnicas en la agricultura e incluso otros productos que se puedan cultivar y ofrecer al mercado, consiguiendo con esto tener una disminución en la producción, y por los altos costos, se produce una desmotivación en la distribución de los productos.

Todas estas causas y consecuencias derivan en el estancamiento de los emprendimientos. El problema central de la presente investigación fue la baja competitividad de la producción agroecológica no industrializadas, que se realizó en cantón Cuenca-Ecuador, por lo que, como objetivo principal fue proponer un modelo matemático en base a la teoría de juegos cooperativos con la intención de mejorar la productividad en la distribución de productos agroecológicos. Un juego cooperativo es un par $(\mathrm{N}, \mathrm{v})$ formado por un conjunto finito $\mathrm{N}=\{1,2, \cdots, \mathrm{n}\}$ y una función $\mathrm{v}: 2^{\mathrm{N}} \rightarrow \mathrm{R}$ que asigna a cada subconjunto $\mathrm{S}$ de $\mathrm{N}$ un número real $\mathrm{v}(\mathrm{S})$ con la condición de que $v(\varnothing)=0$ (Peleg y Sudhölter, 2007).

Cada elemento del conjunto $\mathrm{N}$ es un jugador y cada subconjunto de $\mathrm{N}$ es una coalición (Young, 2005). La función $\mathrm{v}$ se llama función característica del juego (v(S) se considera una medida de la expectativa de la coalición S) y en los casos en los que no hay ambigüedad, se habla del juego $\mathrm{v}$ sobreentendiéndose el conjunto de jugadores Cabrera et al. (2019). Acorde a la teoría de juegos, una coalición es una agrupación o asociación de sujetos con un único fin, que es el de mejorar su utilidad, matemáticamente se diría que es un conjunto $\mathrm{N}$ de $\mathrm{n}$ individuos (Granot, 2010) que conforman las $2^{\mathrm{N}}$ coaliciones (Jiménez-Losada, 2017).

Se realizó un estudio con enfoque mixto, ya que en la parte cuantitativa se utilizaron encuestas semiestructuradas, análisis descriptivo, así como información secundaria para el modelo matemático numérico de la teoría de juegos cooperativos. En la parte cualitativa, al ser un fenómeno poco estudiado, el tamaño de la muestra fue pequeño y el muestreo fue por conveniencia. Por su parte, el diseño de investigación fue no experimental, transversal ya que los datos se recolectaron en un solo momento (2019) (Hernández-Sampieri y Mendoza-Torres, 2018).

\section{Región de estudio}

El cantón Cuenca es la capital de la provincia del Azuay, siendo ésta la tercera provincia más importante del Ecuador y está conformada por 22 parroquias rurales y 15 urbanas. Según el Plan de Ordenamiento Territorial del municipio de Cuenca $65.6 \%$ de la población se concentra en el 
área urbana del cantón y el restante $34.4 \%$ se encuentra en el área rural (Sistema Nacional de Información, 2019). En Azuay se ha generado la producción de nuevos productos, gracias a la ubicación geográfica la cual ha sido favorable; no obstante, en los mercados de comercialización del Azuay existe una baja presencia de productos agroecológicos, debido a la poca información que la población tiene acerca de los mismos.

Los agricultores han podido mejorar su producción con el apoyo del MAGAP para producir de forma agroecológica; sin embargo, la incertidumbre de los productores por falta de control de precios, para la venta de sus productos como para la adquisición de materia prima e insumos necesarios para la producción, provoca que los productores prefieran dedicarse a otras actividades (Rivera, 2019).

\section{Marco muestral}

Para la obtención de los puntos de localización de los agroproductores de la región de estudio se usó un GPS Garmin 64 x, posteriormente con el software online ArcGeek se realiza el cambio de coordenadas geográficas a coordenadas UTM, logrando conseguir valores en (x, y). Una vez definidos los puntos georreferenciados, se definen las características de los productores, con sus respectivas restricciones particulares al caso, para poderlos encuestar.

\section{Tamaño de la muestra y muestreo}

Para analizar el caso de estudio, se buscó la zona que tiene mayor productividad agrícola y que no trabajan en asociación, de los cuales mediante la técnica bola de nieve se pudo ir contactando de uno en uno a los diferentes productores llegando a obtener la información de 76 agroproductores, se les contactó presencialmente en los meses de octubre, noviembre y diciembre de 2019 a primeras horas de la mañana, en vista de que era el horario que disponían de tiempo. El muestreo fue por conveniencia, debido a que los productores no pertenecían a una asociación; mediante la técnica bola de nieve se contactó a un agroproductor, mismo que brindó la información de otro agroproductor y así sucesivamente hasta completar los 76.

\section{Recopilación de información primaria}

Sujetos de estudio. Hay dos tipos, los que pertenecen y los que no a una asociación. La investigación se llevó a cabo con 76 agroproductores que trabajan no asociados y que pertenecen a las parroquias rurales del cantón Cuenca, Ecuador.

Instrumento de medición. Se aplicó una encuesta semiestructurada de 13 ítems, considerando las siguientes temáticas: pertenencia a una asociación, nombre y estatus de legalidad de la asociación, espacio de terreno destinado para el cultivo, lugares de comercialización de sus productos, tipo de producto que cultiva, tipo de producto que comercializa, costo de los productos, cantidad de productos, frecuencia de venta, disponibilidad de transporte, costo de comercialización, agrupación para la comercialización, número de personas que se agrupan para comercializar, además de su ubicación geográfica, edad, género, nivel de estudios y el número de dependencias económicas. 
La información de la encuesta se analizó mediante la estadística descriptiva (frecuencias, porcentajes y promedios). Finalmente, mediante el software Matlab se simula dos escenarios comparativos de trabajar o no en asociación. El periodo de estudio comprendió el año 2019.

\section{Información secundaria}

Para modelar mediante la teoría de juegos cooperativos con utilidad transferible se utilizó las distancias de los productores, la oferta y demanda potencial.

Variable distancia. Uno de los parámetros necesarios para el planteamiento del modelo de teoría de juegos es la distancia; para esto, se tomó como punto (0.0), al centro de la parroquia, sus coordenadas geográficas: [-2.8951084, 79.0512559], UTM [x, y]: [716613.3, 9679814.7], cada nuevo valor UTM de los productores agroecológicos levantados en la encuesta se resta al valor UTM del centro y posteriormente, para no trabajar con valores grandes en la simulación, se procede a realizar una división sobre 1 000. Una vez que obtenemos los resultados de la simulación realizamos el proceso inverso hasta conseguir valores en coordenadas geográficas y proceder a ubicar en el mapa.

Variables oferta y demanda. En base a los datos estadísticos del último Censo de Población y Vivienda del Ecuador realizado en 2010 por el Instituto Nacional de Estadísticas y Censos (INEC), Cuenca está conformada por 22 parroquias rurales y 15 urbanas, para efecto de dicho estudio se tomó en cuenta las 15 parroquias urbanas del cantón y según el Plan de Ordenamiento Territorial del municipio de Cuenca, $65.6 \%$ de la población se concentra en el área urbana del cantón y el restante 34.3\% se encuentra en el área rural (Gobierno Autónomo Descentralizado Municipal del Cantón Cuenca, 2019), además, la población económicamente activa del cantón Cuenca se concentra sobre todo en el área urbana con 69\% (Alcaldía de Cuenca, 2016); es decir, que en la parte urbana, es en donde se ubica mayormente la zona comercial.

El mercado potencial para las ferias agroecológicas son todas las personas (209 245) que viven en la ciudad de Cuenca entre edades de 18 y 70 años que consumen productos agrícolas, siendo este segmento de personas quienes cuenta con el poder adquisitivo para el consumo de estos productos (Instituto Nacional de Estadísticas y Censos, 2018).

\section{Perfil de los agro-productores}

Los resultados de las encuestas nos indican que, en lo relacionado a la edad y el género, $76 \%$ de personas que se dedican al sector agrícola son mujeres frente a $24 \%$ que lo hacen los hombres, con edades desde los 18 años que están en las labores del campo. En el nivel de educación $67 \%$ tienen solo educación primaria. El $67 \%$ pertenecen a una asociación legalmente constituida, de los cuales hacen uso del suelo como sociedad legal apenas 6.1\% (13 $331 \mathrm{ha})$. El 1\% de los productores realizan la comercialización en el lugar de producción (gobiernos autónomos descentralizados), los demás prefieren ir a los mercados mayoristas para su comercialización, lo que conlleva a analizar las variables distancia y costo.

Los tipos más representativos de productos que se cultivan son frutas, hortalizas y verduras, cárnicos y quesos de los cuales, las legumbres, hortalizas y frutas son los que más se comercializa. Los precios de los productos tienen una referencia a una unidad de dólar, viéndolo como un valor constante para la simulación. Del análisis de la oferta se determina que el promedio de 
comercialización es de $2271 \mathrm{~kg}$, de igual manera el tiempo de comercialización es cada semana, los productos son llevados a su comercialización en su gran mayoría a través del alquiler, cuyo costo está en función de la distancia en un valor de 5 USD, en cuyo caso no trabajan de forma organizada y cada uno usa un medio de transporte individual.

La obtención de las variables más significativas en lo relacionados a los productos de oferta y demanda se obtuvo de la encuesta donde las hortalizas, verduras y las frutas predominan con $36.8 \%$, seguido de frutas con un 25.8, granos con 6.9\%, hierbas medicinales $4.9 \%$, lácteos $4.9 \%$, cárnicos $3.6 \%$, huevos $2.6 \%$, animales menores $1.7 \%$ y otros $0.4 \%$. De la misma manera los agroproductores trabajan en base a cadenas cortas de valor, que según Santacoloma (2016), es la proximidad entre consumidores y productores. En este caso específico se identificó que los productores comercializan sus productos en ferias locales o mercados mayoristas del cantón.

\section{Datos de entrada de la simulación utilizando la teoría de juegos}

Con los datos de la oferta, demanda, costo, lugares de comercialización y georreferenciación obtenidos de la encuesta se diseñó la simulación utilizando la teoría de juegos con utilidad transferible, adicionalmente la variable amortización $(\mathrm{R}=0.6)$ fue considerado el costo de depreciación del uso del suelo, productos $(30 \mathrm{~kg}$ ), transporte en un periodo de 365 días, los productos mercado y agroecológicos, son el promedio de la cantidad de productos que producen en los sectores rurales; la distancia umbral $(\mathrm{Du}=3.8 \mathrm{~km})$, es la distancia máxima de comercialización.

La ubicación (0.0), es considerado el centro de la parroquia rural, en base a esta referencia se obtiene la ubicación geográfica (distancia) de cada productor, así como, la oferta (2 $271 \mathrm{~kg}$ ), demanda y precios $\left(4 \$ \mathrm{~kg}^{-1}\right)$, mismos que permiten calcular la constante de pérdidas (0.5). La constante de pérdidas hace referencia al promedio de la diferencia de precios entre el productor agroecológico y un mercado mayorista o centro comercial.

\section{Datos salida de la simulación}

Al aplicar el modelo matemático se obtiene que el promedio de pérdidas sin asociarse es de 15.4 (kg) de productos, representando 53.7\%. Esto se da, en función que la mayoría de los productores ofertan los mismos productos y empiezan a competir entre ellos, en tal sentido, es necesario que trabajen y se constituyan en asociación, pero que lo hagan de forma técnica, considerando las variables antes trabajadas.

De las simulaciones obtenemos, que, de los 76 productores, se deben conformar 8 asociaciones principales [productor $\mathrm{x}$ - productor $\mathrm{y}$ ]: [1 - 15], [17 - 38], [18 - 3], [19 - 33], [20 - 24], [21 - 54], [22 - 10] y [23 - 11].

A los demás productores, se les recomienda que deben mejorar la oferta y buscar otra demanda en los diferentes puntos de comercialización de la ciudad de Cuenca. Una vez que se realicen las primeras ocho asociaciones de dos agroproductores (Figura 1), se reduce a $14.4 \mathrm{~kg}$ de pérdidas, lo que significa que con estas asociaciones se mejora $6.4 \%$ la productividad. 


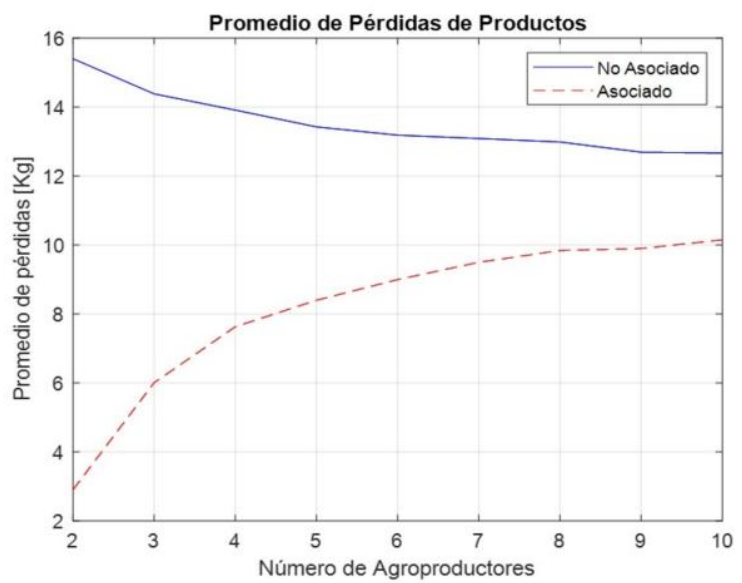

Figura 1. Comparativo de pérdidas de productos al trabajar o no asociados en Cuenca-Ecuador.

Adicionalmente, se observa que a medida que se incrementa el número de productores, las pérdidas también se incrementan, justamente por la competencia interna existente entre los productores. Esto implicaría que tampoco se pueden unir todos los productores y generar una gran asociación, con las condiciones actuales, en vista de que su competencia será mayor y por ende las pérdidas (Figura 2).

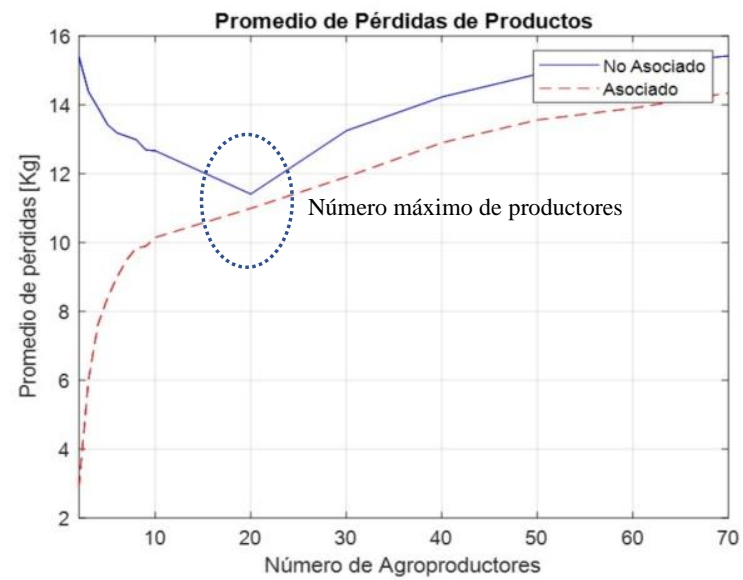

Figura 2. Promedio de pérdidas de los agroproductores estudiados en Cuenca-Ecuador.

\section{Conclusiones}

En base a los resultados de la simulación, se pudo comprobar que los 76 agroproductores no se encuentran técnicamente bien agrupados, actualmente prefieren trabajar de forma aislada, debido a discrepancias internas con la administración, demasiados trámites para su conformación y le ven como una pérdida de tiempo las reuniones pudiendo ser por la relación de que $67 \%$ tienen solo educación primaria. El modelo planteado indica que se debe conformar 8 asociaciones, lo que permitirá mejorar un $6.4 \%$ en la productividad. 
Estas asociaciones deberían ser de un número máximo de 20 agroproductores, de los cuales 10 vienen a ser el valor óptimo porque a medida que se incrementa el número de productores dentro de una asociación, estas pérdidas se incrementan similar a lo que asevera la ley de los rendimientos decrecientes (Brown, 1984) esto es que cuando aumenta la cantidad de insumo variable (número de productores) a una unidad fija (la asociación ya sea en sus instalaciones o infraestructura), los rendimientos empiezan a decrecer.

Para mejorar la productividad de los productores es necesario trabajar en asociación, tanto para conseguir recursos económicos, así como para poder acceder a capacitaciones, pero es necesario analizar la forma en la que deberían asociarse técnicamente. Se debe administrar de mejor manera los productos, el uso del suelo, buscando no tener una competitividad interna entre ellos. Si bien, la producción de sus productos en un gran porcentaje es para el autoconsumo, se debe considerar como trabajos futuros complementarios a la investigación, las capacidades blandas, el tener un plan de siembra dentro de la asociatividad, logrando gestionar el tiempo y los recursos humanos.

\section{Literatura citada}

Altier, M.; Nicholls, C. and Henao, A. Y. 2015. Agroecology and the design of climate changeresilient farming system. Agron. Sustainable Development. 35:869-890.

Altieri, M. A. 2002. Agroecology: the science of natural resource management for poor farmers in marginal environments. Agric. Ecosyst. Environ. (Ed.). 1-24 pp.

Altieri, M. and Toledo, V. M. 2011. The agroecological revolution of latin America: rescuing nature, securing food sovereignity and empowering peasants. J. Peasant Studies. 587-612 pp.

Barkin, D. y Fuentes-Carrasco, M. Z. 2012. La significación de una economía ecológica radical. Rev. Iberoam. Econ. Ecol. 19:1-14.

Brown, F. 1984. La evolución de la teoría de la oferta en condiciones de competencia perfecta. Inv. Econ. 43(167):255-258. http://www.jstor.org/stable/42779424.

Cabrera, J.; Morales, D. and Medina, R. 2019. Reducing power losses in smart grids with cooperative game theory. In: Ustun, T. S. Advanced communication and control methods for future Smartgrids London, United Kingdom. Intechopen. 49-65 pp.

Gobierno Autónomo Descentralizado Municipal del Cantón Cuenca. 2019. Alcaldía de Cuenca. http://www.cuenca.gov.ec/.

Granot, D. 2010. The reactive bargaining set for cooperative games. Inter. J. Game Theory. 39(1):163-170.

Hair, J. B. 2004. Investigación de mercados. McGraw-Hill. México, DF.

Heifer. 2016. Plan de negocios feria agroecológica de la escuela agroecológica de mujeres Saraguras. http://www.heiferecuador.org/wp-content/uploads/2018/03/5.-plan-de-negocios feria-eams.pdf.

Hernández-Sampieri, R. y Mendoza-Torres, C. P. 2018. Metodología de la investigación. Las rutas cuantitativa, cualitativa y mixta. Mc Graw Hill Education. México, DF.

Instituto Nacional de Estadísticas y Censos. 2018. Ecuador en cifras. https://www.ecuadorencifras. gob.ec/encuesta-de-superficie-y-produccion-agropecuaria-continua-2018/.

Intriago, R. Y. 2018. Agroecología en el ecuador. Proceso histórico, logros y desafíos. Agroecología. 11(2):95-113.

Jiménez-Losada, A. 2017. Models for cooperative games with fuzzy relations among the agents. Studies in Fuzziness Soft Computing. 355:1-36. 
MAG. 2014. Ministerio de agricultura y ganadería la agroecología está presente: mapeo de productores agroecológicos y del estado de la agroecología en la sierra y costa ecuatoriana. Quito: Heifer-Ecuador.

Peleg, B. and Sudhölter, P. 2007. Introduction to the theory of cooperative games. Berlín: Springer, Berlin, Heidelberg.

Rivera, C. 2019. Desarrollo de estrategias para el incremento de consumo de productos agroecológicos en la provincia del Azuay-Ecuador. Buenos Aires.

Saal, M. Y. 2015. Agroecología y agroecología y soberanía alimentaria, el caso de la feria agroecológica de Córdoba. https://issuu.com/magdalenasaal/docs/merged?fbclid= iwar0x urguxz-bzskbmujloi_ho81-yljtkbsj7ol21lnk1wjhebejeszr4do.

Santacoloma, P. 2016. Hacia una definición de cadenas cortas agroalimentarias. Taller de intercambio de experiencias: cadenas cortas agroalimentarias. FAO. Ciudad de México. 593dp.com/index.php/593_digital_publisher/login/signIn. 5-7 pp.

SIN. 2019. Sistema Nacional de Información. 2019. Planes de desarrollo y ordenamiento territorial. Retrieved from https://multimedia.planificacion.gob.ec/pdot/descargas.html.

Toledo, V. M.; Boege, E. and Barrera-Bassol, N. 2010. The biocultural heritage of México: an overview. Landscape. (Ed.). 6-10 pp.

Young, H. 2005. Monotonic solutions of cooperative game. Inter. J. Game Theory. 14:65-72. 